PROCEEDINGS OF THE AMERICAN MATHEMATICAL SOCIETY

Volume 125, Number 4, April 1997, Pages 1011-1017

S 0002-9939(97)03923-3

\title{
APPLICATIONS OF RESIDUES TO COMBINATORIAL IDENTITIES
}

\author{
I-CHIAU HUANG
}

(Communicated by Wolmer V. Vasconcelos)

\begin{abstract}
A concrete aspect of Grothendieck Duality is used to give local cohomology proofs of combinatorial identities including MacMahon's master theorem, Grosswald identity, identity of Shoo, Tepper identity, and others.
\end{abstract}

In combinatorial analysis, the method of integral representation is widely used (see, for example, [1]). This analytic method provides an analytic framework in which many combinatorial problems can be systematically solved. The basis for this method is given with the help of the formalism of residues and the formal properties for transforming integrals. The symbolic apparatus of this method dates back at least to 18th century, however, without rigorous foundations. For instance, the convergence of series was not taken into account.

On the other hand, the method of formal power series and formal Laurent series has also been developed for solving combinatorial problems (see, for example, [3]). Contour integrals in the analytic method are replaced by "taking the coefficient of a monomial in a series" in this algebraic method. However the concept of differentials is lacking in the algebraic side. In this article, we enrich the existing algebraic methods by using a concrete aspect of Grothendieck Duality. "Taking the coefficient" thus becomes more natural with the presence of differentials.

In section 1, we give an algebraic definition of residues and review their properties. In section 2 , we give local cohomology proofs of some known combinatorial identities which were shown by the analytic methods in [1] and [2]. Our proofs resemble the symbolic guise of the proofs in [1] or [2]. For the general language of commutative algebra, the reader is referred to [7], [5], and [4].

\section{Residues}

Let $\kappa$ be a field. Formal power series rings over $\kappa$ can be characterized without referring to variables. Assume that a Noetherian local ring $(R, \mathfrak{m})$ containing $\kappa$ is a formal power series ring of $n$ variables over $\kappa$, that is, the following three conditions are satisfied.

- $R$ is complete and is of Krull dimension $n$.

- $R$ is $\mathfrak{m}$-smooth over $\kappa$.

- The induced map $\kappa \rightarrow R / \mathfrak{m}$ is an isomorphism.

Received by the editors October 31, 1995.

1991 Mathematics Subject Classification. Primary 05A19; Secondary 13F25.

Key words and phrases. Combinatorial identity, residue.

(C)1997 American Mathematical Society 
Choose a regular system of parameters $X_{1}, \cdots, X_{n}$ of $R$ (that is, $n$ elements which generate $\mathfrak{m})$. Then elements in $R$ can be written uniquely as an infinite sum

$$
\sum_{i_{1}, \cdots, i_{n} \geq 0} a_{i_{1}, \cdots, i_{n}} X_{1}^{i_{1}} \cdots X_{n}^{i_{n}}, \quad a_{i_{1}, \cdots, i_{n}} \in \kappa .
$$

The universal separated differential module $\left(\tilde{\Omega}_{R / \kappa}, d\right)$ of $R$ over $\kappa$ is freely generated by $d X_{1}, \cdots, d X_{n}$. Elements in the top local cohomology module $\mathrm{H}_{\mathfrak{m}}^{n}\left(\wedge^{n} \tilde{\Omega}_{R / \kappa}\right)$ of $\wedge^{n} \tilde{\Omega}_{R / \kappa}$ supported at $\mathfrak{m}$ can be described using generalized fractions:

Definition 1. Let $f_{1}, \cdots, f_{n}$ be a system of parameters of $R$ (that is, $n$ elements which generate $\mathfrak{m}$ up to radical). We define

$$
\left[\begin{array}{c}
\omega \\
f_{1}^{i_{1}}, \cdots, f_{n}^{i_{n}}
\end{array}\right]_{f_{1}, \cdots, f_{n}} \quad\left(\omega \in \wedge^{n} \tilde{\Omega}_{R / \kappa}, i_{1}, \cdots, i_{n}>0\right)
$$

to be the image of

$$
\frac{f_{1}^{i-i_{1}} \cdots f_{n}^{i-i_{n}} \omega}{\left(f_{1} \cdots f_{n}\right)^{i}} \quad\left(i=i_{1}+\cdots+i_{n}\right)
$$

under the canonical map

$$
\left(\wedge^{n} \tilde{\Omega}_{R / \kappa}\right)_{f_{1} \cdots f_{n}} \rightarrow \mathrm{H}_{\mathfrak{m}}^{n}\left(\wedge^{n} \tilde{\Omega}_{R / \kappa}\right) .
$$

Theorem 1. If $g_{1}=f_{1}^{i_{1}}, \cdots, g_{n}=f_{n}^{i_{n}}$, then

$$
\left[\begin{array}{c}
\omega \\
f_{1}^{i_{1}}, \cdots, f_{n}^{i_{n}}
\end{array}\right]_{f_{1}, \cdots, f_{n}}=\left[\begin{array}{c}
\omega \\
f_{1}^{i_{1}}, \cdots, f_{n}^{i_{n}}
\end{array}\right]_{g_{1}, \cdots, g_{n}} .
$$

A proof of the above theorem is sketched in $[4,(2.2)]$. As a consequence of the above theorem, we may write generalized fractions without subscript. Generalized fractions enjoy the following properties:

Property 1 (linearity law). For $\omega_{1}, \omega_{2} \in \wedge^{n} \tilde{\Omega}_{R / \kappa}, i_{1}, \cdots, i_{n}>0$, and $k_{1}, k_{2} \in \kappa$,

$$
\left[\begin{array}{c}
k_{1} \omega_{1}+k_{2} \omega_{2} \\
f_{1}^{i_{1}}, \cdots, f_{n}^{i_{n}}
\end{array}\right]=k_{1}\left[\begin{array}{c}
\omega_{1} \\
f_{1}^{i_{1}}, \cdots, f_{n}^{i_{n}}
\end{array}\right]+k_{2}\left[\begin{array}{c}
\omega_{2} \\
f_{1}^{i_{1}}, \cdots, f_{n}^{i_{n}}
\end{array}\right] .
$$

Property 2 (vanishing law; [4], 2.3.i, or [6], 7.2.a). For $\omega \in \wedge^{n} \tilde{\Omega}_{R / \kappa}$,

$$
\left[\begin{array}{c}
\omega \\
f_{1}^{i_{1}}, \cdots, f_{n}^{i_{n}}
\end{array}\right]=0
$$

if and only if $\left(f_{1}^{i_{1}} \cdots f_{n}^{i_{n}}\right)^{s} \omega \in\left(f_{1}^{i_{1}(s+1)}, \cdots, f_{n}^{i_{n}(s+1)}\right) \wedge^{n} \tilde{\Omega}_{R / \kappa}$ for some $s \geq 0$.

In particular, for any $1 \leq j \leq n$,

$$
\left[\begin{array}{c}
f_{j}^{i_{j}} \omega \\
f_{1}^{i_{1}}, \cdots, f_{n}^{i_{n}}
\end{array}\right]=0
$$

Property 3 (transformation law; [4], 2.3.ii, or [6], 7.2.b). For $\omega \in \wedge^{n} \tilde{\Omega}_{R / \kappa}$ and a system of parameters $f_{1}^{\prime}, \cdots, f_{n}^{\prime}$ of $R$,

$$
\left[\begin{array}{c}
\omega \\
f_{1}, \cdots, f_{\ell}
\end{array}\right]=\left[\begin{array}{c}
\operatorname{det}\left(r_{i, j}\right) \omega \\
f_{1}^{\prime}, \cdots, f_{\ell}^{\prime}
\end{array}\right]
$$

if $f_{i}^{\prime}=\sum_{j=1}^{n} r_{i, j} f_{j}$ for $i=1, \cdots, n$. 
Note that elements in $\mathrm{H}_{\mathfrak{m}}^{n}\left(\wedge^{n} \tilde{\Omega}_{R / \kappa}\right)$ can be written uniquely as a finite sum

$$
\sum_{i_{1}, \cdots, i_{n}>0}\left[\begin{array}{c}
a_{i_{1}, \cdots, i_{n}} d X_{1} \cdots d X_{n} \\
X_{1}^{i_{1}}, \cdots, X_{n}^{i_{n}}
\end{array}\right], \quad a_{i_{1}, \cdots, i_{n}} \in \kappa
$$

So we can make the following definition.

Definition 2. The residue map

$$
\operatorname{res}_{X_{1}, \cdots, X_{n}}: H_{\mathfrak{m}}^{n}\left(\wedge^{n} \tilde{\Omega}_{R / \kappa}\right) \rightarrow \kappa
$$

is defined to be the $\kappa$-linear map satisfying

$$
\operatorname{res}_{X_{1}, \cdots, X_{n}}\left[\begin{array}{cl}
a_{i_{1}, \cdots, i_{n}} d X_{1} \cdots d X_{n} \\
X_{1}^{i_{1}}, \cdots, X_{n}^{i_{n}}
\end{array}\right]= \begin{cases}a_{i_{1}, \cdots, i_{n},}, & \text { if } i_{1}=\cdots=i_{n}=1, \\
0, & \text { otherwise. }\end{cases}
$$

Many of our applications are based on the following theorem which is a special case of $[4,(5.3)]$.

Theorem 2. Let $Y_{1}, \cdots, Y_{n}$ be another regular system of parameters of $R$. Then $\operatorname{res}_{X_{1}, \cdots, X_{n}}=\operatorname{res}_{Y_{1}, \cdots, Y_{n}}$.

So we can write res $X_{1}, \cdots, X_{n}$ simply as res.

Using modules of zero dimensional support, the notion of residues can be extended to power series rings over a complete local ring [4, Chapter 5]. In such general context, residue maps are transitive. Here we only state a special case which will be used later.

Property 4 (transitivity law). For $f_{0}, f_{1}, f_{2} \cdots \in \kappa[[X]]$,

$$
\operatorname{res}_{X, Y}\left[\begin{array}{c}
\left(f_{0}+f_{1} Y+f_{2} Y^{2}+\cdots\right) d X d Y \\
X^{n}, Y^{m}
\end{array}\right]=\operatorname{res}_{X}\left[\begin{array}{c}
f_{m-1} d X \\
X^{n}
\end{array}\right] .
$$

\section{Applications}

Example 1 (MacMahon's Master Theorem). Let $\kappa$ be a field, let

$$
X_{i}=\sum_{j=1}^{n} a_{i j} x_{j} \quad\left(a_{i j} \in \kappa, i=1,2, \cdots, n\right),
$$

and let $m_{1}, m_{2}, \cdots, m_{n}$ be non-negative integers. Then the coefficient of $x_{1}^{m_{1}} \cdots x_{n}^{m_{n}}$ in $X_{1}^{m_{1}} \cdots X_{n}^{m_{n}}$ is equal to the coefficient of $w_{1}^{m_{1}} \cdots w_{n}^{m_{n}}$ in the inverse of

$$
D=\left|\begin{array}{cccc}
1-a_{11} w_{1} & -a_{12} w_{1} & \cdots & -a_{1 n} w_{1} \\
-a_{21} w_{2} & 1-a_{22} w_{2} & \cdots & -a_{2 n} w_{2} \\
\vdots & \vdots & \ddots & \vdots \\
-a_{n 1} w_{n} & -a_{n 2} w_{n} & \cdots & 1-a_{n n} w_{n}
\end{array}\right| .
$$

Proof (cf. [2]). Consider the powers series ring $\kappa\left[\left[x_{1}, \cdots, x_{n}\right]\right]$ in which the theorem is properly stated. Let $Y_{i}=1+X_{i}$. Then the required coefficient is equal to

$$
I=\operatorname{res}\left[\begin{array}{c}
Y_{1}^{m_{1}} \cdots Y_{n}^{m_{n}} d x_{1} \cdots d x_{n} \\
x_{1}^{m_{1}+1}, \cdots, x_{n}^{m_{n}+1}
\end{array}\right] .
$$

Let $w_{i}=x_{i} / Y_{i}(i=1, \cdots, n)$. Then $w_{1}, \cdots, w_{n}$ is a regular system of parameters of $\kappa\left[\left[x_{1}, \cdots, x_{n}\right]\right]$. By the transformation law, we have

$$
I=\operatorname{res}\left[\begin{array}{c}
\left(Y_{1} \cdots Y_{n}\right)^{-1} d x_{1} \cdots d x_{n} \\
w_{1}^{m_{1}+1}, \cdots, w_{n}^{m_{n}+1}
\end{array}\right] .
$$


Since

$$
d w_{1} \cdots d w_{n}=\frac{1}{Y_{1} \cdots Y_{n}}\left|\begin{array}{cccc}
1-a_{11} w_{1} & -a_{12} w_{1} & \cdots & -a_{1 n} w_{1} \\
-a_{21} w_{2} & 1-a_{22} w_{2} & \cdots & -a_{2 n} w_{2} \\
\vdots & \vdots & \ddots & \vdots \\
-a_{n 1} w_{n} & -a_{n 2} w_{n} & \cdots & 1-a_{n n} w_{n}
\end{array}\right| d x_{1} \cdots d x_{n}
$$

we get

$$
I=\operatorname{res}\left[\begin{array}{c}
D^{-1} d w_{1} \cdots d w_{n} \\
w_{1}^{m_{1}+1}, \cdots, w_{n}^{m_{n}+1}
\end{array}\right]
$$

which is the coefficient of $w_{1}^{m_{1}} \cdots w_{n}^{m_{n}}$ in $D^{-1}$.

Let $\kappa$ be a field of characteristic 0 . Consider the power series rings $\kappa[[x]]$ and $\kappa[[x, y]]$. The facts that

$$
\operatorname{res}\left[\begin{array}{c}
(1+x)^{n} d x \\
x^{k+1}
\end{array}\right]=\left(\begin{array}{l}
n \\
k
\end{array}\right)
$$

and

$$
\operatorname{res}\left[\begin{array}{c}
(1+x)^{m}(1+y)^{n} d x d y \\
x^{h+1}, y^{k+1}
\end{array}\right]=\left(\begin{array}{c}
m \\
h
\end{array}\right)\left(\begin{array}{l}
n \\
k
\end{array}\right)
$$

will be used.

Example 2 (cf. [1], (2.1)).

$$
\sum_{k=0}^{q}(-1)^{k}\left(\begin{array}{l}
p \\
k
\end{array}\right)=(-1)^{q}\left(\begin{array}{c}
p-1 \\
q
\end{array}\right), \quad \quad p>q .
$$

Proof.

$$
\begin{aligned}
& \sum_{k=0}^{q}(-1)^{k}\left(\begin{array}{l}
p \\
k
\end{array}\right) \\
= & \sum_{k=0}^{q}(-1)^{k} \operatorname{res}\left[\begin{array}{c}
(1+x)^{p} d x \\
x^{k+1}
\end{array}\right] \\
= & \sum_{k=0}^{q}(-1)^{k} \operatorname{res}\left[\begin{array}{c}
(1+x)^{p} x^{p-k} d x \\
x^{p+1}
\end{array}\right] \quad \text { (transformation law) } \\
= & \operatorname{res}\left[\begin{array}{c}
(1+x)^{p} \sum_{k=0}^{q}(-1)^{k} x^{p-k} d x \\
x^{p+1}
\end{array}\right] \quad \text { (linearity law) } \\
= & \operatorname{res}\left[\begin{array}{c}
(1+x)^{p-1}\left(x^{p+1}+(-1)^{q} x^{p-q}\right) d x \\
x^{p+1}
\end{array}\right] \quad \text { (transformation law) } \\
= & \operatorname{res}\left[\begin{array}{c}
(1+x)^{p-1}(-1)^{q} x^{p-q} d x \\
x^{p+1}
\end{array}\right] \\
= & \operatorname{res}\left[\begin{array}{c}
(-1)^{q}(1+x)^{p-1} d x \\
x^{q+1}
\end{array}\right] \\
= & (-1)^{q}\left(\begin{array}{c}
p-1 \\
q
\end{array}\right) .
\end{aligned}
$$


Example 3 (Grosswald identity; cf. [1], (1.42)). Assume $n-r \equiv 0(\bmod 2)$.

$$
\sum_{\nu=0}^{n-r}(-2)^{-\nu}\left(\begin{array}{c}
n \\
r+\nu
\end{array}\right)\left(\begin{array}{c}
n+r+\nu \\
\nu
\end{array}\right)=(-1)^{(n-r) / 2} 2^{r-n}\left(\begin{array}{c}
n \\
(n-r) / 2
\end{array}\right) .
$$

Proof. Replacing $\nu$ by $n-r-\nu$, we have

$$
\sum_{\nu=0}^{n-r}(-2)^{-\nu}\left(\begin{array}{c}
n \\
r+\nu
\end{array}\right)\left(\begin{array}{c}
n+r+\nu \\
\nu
\end{array}\right)=\sum_{\nu=0}^{n-r}(-2)^{r-n+\nu}\left(\begin{array}{l}
n \\
\nu
\end{array}\right)\left(\begin{array}{c}
2 n-\nu \\
n-r-\nu
\end{array}\right)
$$

Using the transformation law and the linearity law, we have

$$
\begin{aligned}
& \sum_{\nu=0}^{n-r}(-2)^{r-n+\nu}\left(\begin{array}{l}
n \\
\nu
\end{array}\right)\left(\begin{array}{c}
2 n-\nu \\
n-r-\nu
\end{array}\right)=2^{r-n} \sum_{\nu=0}^{n-r} \operatorname{res}\left[\begin{array}{c}
(-2)^{\nu}\left(\begin{array}{c}
n \\
\nu
\end{array}\right)(1+x)^{2 n-\nu} d x \\
x^{n-r-\nu+1}
\end{array}\right] \\
& =2^{r-n} \sum_{\nu=0}^{n-r} \operatorname{res}\left[\begin{array}{c}
\left(\begin{array}{l}
n \\
\nu
\end{array}\right)(1+x)^{2 n-\nu}(-2 x)^{\nu} d x \\
x^{n-r+1}
\end{array}\right]
\end{aligned}
$$

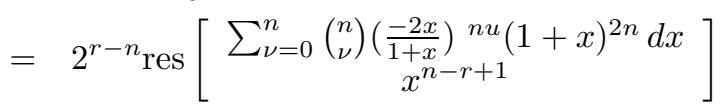

$$
\begin{aligned}
& =2^{r-n} \operatorname{res}\left[\begin{array}{c}
\left(1-\frac{2 x}{1+x}\right)^{n}(1+x)^{2 n} d x \\
x^{n-r+1}
\end{array}\right] \\
& =2^{r-n} \operatorname{res}\left[\begin{array}{c}
\left(1-x^{2}\right)^{n} d x \\
x^{n-r+1}
\end{array}\right] \text {. }
\end{aligned}
$$

Using the linearity law and the definition of residues, we have

$$
2^{r-n} \text { res }\left[\begin{array}{c}
\left(1-x^{2}\right)^{n} d x \\
x^{n-r+1}
\end{array}\right]=(-1)^{(n-r) / 2} 2^{r-n}\left(\begin{array}{c}
n \\
(n-r) / 2
\end{array}\right) .
$$

Example 4 (Identity of Shoo; cf. [1], (2.16)).

$$
\sum_{k=0}^{m}\left(\begin{array}{c}
m \\
k
\end{array}\right)^{2}\left(\begin{array}{c}
n+2 m-k \\
2 m
\end{array}\right)=\left(\begin{array}{c}
m+n \\
n
\end{array}\right)^{2} .
$$

Proof. Using the transformation law and the linearity law, we have

$$
\begin{aligned}
& \sum_{k=0}^{m}\left(\begin{array}{c}
m \\
k
\end{array}\right)^{2}\left(\begin{array}{c}
n+2 m-k \\
2 m
\end{array}\right) \\
& =\sum_{k=0}^{m} \operatorname{res}\left[\begin{array}{c}
\left(\begin{array}{c}
m \\
k
\end{array}\right)(1+x)^{m}(1+y)^{n+2 m-k} d x d y \\
x^{m-k+1}, y^{2 m+1}
\end{array}\right] \\
& =\operatorname{res}\left[\begin{array}{c}
\sum_{k=0}^{m}\left(\begin{array}{c}
m \\
k
\end{array}\right)(1+y)^{m-k} x^{k}(1+x)^{m}(1+y)^{n+m} d x d y \\
x^{m+1}, y^{2 m+1}
\end{array}\right] \\
& =\operatorname{res}\left[\begin{array}{c}
(1+x+y)^{m}(1+x)^{m}(1+y)^{n+m} d x d y \\
x^{m+1}, y^{2 m+1}
\end{array}\right] .
\end{aligned}
$$


Using the transitivity law, the linearity law, and the definition of residues, we have

$$
\begin{aligned}
& \operatorname{res}\left[\begin{array}{c}
(1+x+y)^{m}(1+x)^{m}(1+y)^{n+m} d x d y \\
x^{m+1}, y^{2 m+1}
\end{array}\right] \\
= & \operatorname{res}\left[\begin{array}{c}
(1+x)^{m} \sum_{k=m}^{2 m}\left(\begin{array}{c}
m+n \\
k
\end{array}\right)\left(\begin{array}{c}
m \\
2 m-k
\end{array}\right)(1+x)^{k-m} d x \\
x^{m+1}
\end{array}\right] \\
= & \sum_{k=m}^{2 m}\left(\begin{array}{c}
k \\
m
\end{array}\right)\left(\begin{array}{c}
m+n \\
k
\end{array}\right)\left(\begin{array}{c}
m \\
2 m-k
\end{array}\right) .
\end{aligned}
$$

Using the methods as before, one can verify easily that

$$
\sum_{k=m}^{2 m}\left(\begin{array}{c}
k \\
m
\end{array}\right)\left(\begin{array}{c}
m+n \\
k
\end{array}\right)\left(\begin{array}{c}
m \\
2 m-k
\end{array}\right)=\left(\begin{array}{c}
m+n \\
n
\end{array}\right) \sum_{k=0}^{m}\left(\begin{array}{c}
m \\
m-k
\end{array}\right)\left(\begin{array}{l}
n \\
k
\end{array}\right)=\left(\begin{array}{c}
m+n \\
n
\end{array}\right)^{2} .
$$

Given an element $f$ in the maximal ideal of $\kappa[[x]]$, we denote

$$
e^{f}:=1+f+\frac{f^{2}}{2 !}+\cdots+\frac{f^{n}}{n !}+\cdots
$$

and

$$
\ln (1+f):=f-\frac{f^{2}}{2}+\frac{f^{3}}{3}-\frac{f^{4}}{4}+\frac{f^{5}}{5}-\cdots
$$

Then

$$
\operatorname{res}\left[\begin{array}{c}
-\ln (1-x) d x \\
x^{n+1}
\end{array}\right]=\frac{1}{n}
$$

and

$$
\operatorname{res}\left[\begin{array}{c}
e^{\alpha x} d x \\
x^{n+1}
\end{array}\right]=\frac{\alpha^{n}}{n !}
$$

for $\alpha \in \kappa$.

Example 5 (Tepper identity; cf. [1], (2.2)). Let $\alpha$ be an element in $\kappa$; then

$$
\sum_{k=0}^{r}(-1)^{k}\left(\begin{array}{l}
r \\
k
\end{array}\right)(\alpha-k)^{p}= \begin{cases}0, & 0 \leq p<r \\
r ! & p=r .\end{cases}
$$

Proof.

$$
\begin{aligned}
\sum_{k=0}^{r}(-1)^{k}\left(\begin{array}{l}
r \\
k
\end{array}\right)(\alpha-k)^{p} & =\operatorname{res}\left[\begin{array}{c}
p ! \sum_{k=0}^{r}(-1)^{k}\left(\begin{array}{c}
r \\
k
\end{array}\right) e^{x(\alpha-k)} d x \\
x^{p+1}
\end{array}\right] \\
& =\operatorname{res}\left[\begin{array}{c}
p !\left(1-e^{-x}\right)^{r} e^{x \alpha} d x \\
x^{p+1}
\end{array}\right] \\
& =\operatorname{res}\left[\begin{array}{c}
p ! e^{x \alpha}\left(\frac{x}{1 !}-\frac{x^{2}}{2 !}+\cdots\right)^{r} d x \\
x^{p+1}
\end{array}\right] \\
& = \begin{cases}0, & 0 \leq p<r \\
r !, & p=r .\end{cases}
\end{aligned}
$$


Example 6 (cf. [1], (2.26)).

$$
\sum_{k=1}^{n}(-1)^{k-1}\left(\begin{array}{l}
n \\
k
\end{array}\right) \sum_{j=1}^{k} \frac{1}{j}=\frac{1}{n} .
$$

Proof.

$$
\begin{aligned}
\sum_{k=1}^{n}(-1)^{k-1}\left(\begin{array}{l}
n \\
k
\end{array}\right) \sum_{j=1}^{k} \frac{1}{j} & =\sum_{k=1}^{n} \sum_{j=1}^{k} \operatorname{res}\left[\begin{array}{c}
(-1)^{k}\left(\begin{array}{l}
n \\
k
\end{array}\right) \ln (1-x) d x \\
x^{j+1}
\end{array}\right] \\
& =\sum_{k=1}^{n} \sum_{j=1}^{k} \operatorname{res}\left[\begin{array}{c}
(-1)^{k}\left(\begin{array}{l}
n \\
k
\end{array}\right) x^{k-j} \ln (1-x) d x \\
x^{k+1}
\end{array}\right] \\
& =\sum_{k=1}^{n} \operatorname{res}\left[\begin{array}{c}
(-1)^{k}\left(\begin{array}{c}
n \\
k
\end{array}\right)\left(1-x^{k}\right) \ln (1-x) d x \\
x^{k+1}(1-x)
\end{array}\right] \\
& =\sum_{k=0}^{n} \operatorname{res}\left[\begin{array}{c}
(-1)^{k}\left(\begin{array}{l}
n \\
k
\end{array}\right) x^{n-k} \ln (1-x) d x \\
x^{n+1}(1-x)
\end{array}\right] \\
& =\operatorname{res}\left[\begin{array}{c}
(x-1)^{n} \ln (1-x) d x \\
x^{n+1}(1-x)
\end{array}\right] \\
& =\operatorname{res}\left[\begin{array}{c}
-\ln (1-w) d w \\
w^{n+1}
\end{array}\right] \\
& =\frac{1}{n} .
\end{aligned}
$$

\section{REFERENCES}

1. G. P. Egorychev. Integral Representation and the Computation of Combinatorial Sums, volume 59 of Translation of Mathematical Monographs. American Mathematical Society, 1984. MR 85a:05008

2. I. J. Good. A short proof of MacMahon's 'master theorem'. Proc. Cambridge Philos. Soc., 58:160, 1962. MR 25:1109a

3. I. P. Goulden and D. M. Jackson. Combinatorial Enumeration. John Wiley \& Sons, 1983. MR 84m:05002

4. I-C. Huang. Pseudofunctors on modules with zero dimensional support. Memoirs of the American Mathematical Society, 114(548), 1995. MR 95h:13013

5. E. Kunz. Kähler Differentials. Vieweg, Braunschweig, Wiesbaden, 1986. MR 88e:14025

6. J. Lipman. Dualizing Sheaves, Differentials and Residues on Algebraic Varieties. Astérisque 117, 1984. MR 86g:14008

7. H. Matsumura. Commutative Ring Theory. Cambridge University Press, 1986. MR 88h:13001

Institute of Mathematics, Academia Sinica, Nankang, Taipei 11529, Taiwan, Republic OF CHINA

E-mail address: ichuang@math.sinica.edu.tw 